\title{
Some Results on the Differential Geometry of Spacelike Curves in De-Sitter Space
}

\author{
Tunahan Turhan ${ }^{1 *}$, Nihat Ayyildiz ${ }^{2}$ \\ ${ }^{1}$ Seydişehir Vocational School, Necmettin Erbakan University, Konya, Turkey \\ ${ }^{2}$ Department of Mathematics, Süleyman Demirel University, Isparta, Turkey \\ Email: "tturhan07@gmail.com, *tturhan@konya.edu.tr, nihatayyildiz@sdu.edu.tr, ayyildiz67@gmail.com
}

Received June 14, 2013; revised July 15, 2013; accepted September 1, 2013

Copyright (C) 2013 Tunahan Turhan, Nihat Ayyildiz. This is an open access article distributed under the Creative Commons Attribution License, which permits unrestricted use, distribution, and reproduction in any medium, provided the original work is properly cited.

\section{ABSTRACT}

The differential geometry of curves on a hypersphere in the Euclidean space reflects instantaneous properties of spherecal motion. In this work, we give some results for differential geometry of spacelike curves in 3-dimensional de-Sitter space. Also, we study the Frenet reference frame, the Frenet equations, and the geodesic curvature and torsion functions to analyze and characterize the shape of the curves in 3-dimensional de-Sitter space.

Keywords: De-Sitter Space; Frenet Equations; Frenet Reference Frame; Geodesic Curvature and Torsion; Local Canonical Form

\section{Introduction}

Let $\mathbb{R}^{4}=\left\{x=\left(x_{1}, x_{2}, x_{3}, x_{4}\right) \mid x_{i} \in \mathbb{R}, 1 \leq i \leq 4\right\}$ be a 4 -dimensional vector space. The Lorentzian space $\left(\mathbb{R}^{4},\langle\rangle,\right)=\mathbb{R}_{1}^{4}$ is the 4 -dimensional vector space $\mathbb{R}^{4}$ endowed with the pseudo scalar product

$$
\langle x, y\rangle=x_{1} y_{1}+x_{2} y_{2}+x_{3} y_{3}-x_{4} y_{4}
$$

where $x=\left(x_{1}, x_{2}, x_{3}, x_{4}\right), \quad y=\left(y_{1}, y_{2}, y_{3}, y_{4}\right), \mathbb{R}_{1}^{4}, \quad$ [1]. The norm of a vector $x \in \mathbb{R}_{1}^{4}$ is defined by $\|x\|=\sqrt{|\langle x, x\rangle|}$. Let $\mathbb{S}_{1}^{3}$ denote the 3-dimensional unitary de-Sitter space, that is, $\mathbb{S}_{1}^{3} \subset \mathbb{R}_{1}^{4}$ is the hyperquadric, $[2,3]$,

$$
\mathbb{S}_{1}^{3}=\left\{x \in R_{1}^{4} \mid\langle x, x\rangle=1\right\} .
$$

Given 3-vector $x=\left(x_{1}, x_{2}, x_{3}, x_{4}\right), y=\left(y_{1}, y_{2}, y_{3}, y_{4}\right)$, $z=\left(z_{1}, z_{2}, z_{3}, z_{4}\right)$, in $\mathbb{R}_{1}^{4}$. Then we can define the wedge product $x \wedge y \wedge z$ as follows

$$
x \wedge y \wedge z=\left|\begin{array}{cccc}
e_{1} & e_{2} & e_{3} & -e_{4} \\
x_{1} & x_{2} & x_{3} & x_{4} \\
y_{1} & y_{2} & y_{3} & y_{4} \\
z_{1} & z_{2} & z_{3} & z_{4}
\end{array}\right|
$$

where $\left\{e_{1}, e_{2}, e_{3}, e_{4}\right\}$ is the canonical basis of $\mathbb{R}_{1}^{4}$, [2].

A spherical displacement can be specified by a unit

\footnotetext{
"Corresponding author.
}

vector $u=\left(u_{x}, u_{y}, u_{z}\right)$ along the axis of the rotation and a rotation angle $\phi$. The Euler parameters of the rotation defined in terms of $u$ and $\phi$ can be used to prescribe a mapping of this rotation to a point in a higher dimensional space [4-6]. The vector function $X(u, \phi)=\left(X_{1}, X_{2}, X_{3}, X_{4}\right)$ is given by

$$
\begin{aligned}
& X_{1}=\cosh \frac{\phi}{2}, X_{2}=\sinh \frac{\phi}{2} u_{x}, \\
& X_{3}=\sinh \frac{\phi}{2} u_{y}, X_{4}=\sinh \frac{\phi}{2} u_{z} .
\end{aligned}
$$

Let $u=\left(u_{x}, u_{y}, u_{z}\right)$ denote a timelike rotation axis. So, we get

$$
\begin{aligned}
& X_{1}^{2}+X_{2}^{2}+X_{3}^{2}-X_{4}^{2} \\
& =\cosh ^{2} \frac{\phi}{2}+\left(u_{x}^{2}+u_{y}^{2}-u_{z}^{2}\right) \sinh ^{2} \frac{\phi}{2}=1 .
\end{aligned}
$$

This means that the point $X$ lies on the hyperquadric of radius 1 in $\mathbb{R}_{1}^{4}$. Let us denote this hyperquadric $\mathbb{S}_{1}^{3}$. Our aim is to give an interpretation of the image of the mapping $X(u, \phi)$. For this, we will examine the differential geometry of curves on $\mathbb{S}_{1}^{3}$. So, we will introduce a Frenet frame for the curve and define the geodesic curvature and geodesic torsion functions which characterize the shape of the curve. Also we will give explicit formulas for the geodesic curvature and torsion functions of the parameterized curve $X(t)$. For this aim, 
we will use the exterior algebra of multivectors.

\section{The Frenet Reference Frame}

Let us consider a general parametrized spacelike curves on $\mathbb{S}_{1}^{3}$ denoted by $X(t)$. We will focus on the geometric properties of $X(t)$. For this, we define arclenght parameter $s$ as

$$
s(t)=\int_{0}^{t}\left\|\frac{\mathrm{d} X}{\mathrm{~d} t}\right\| \mathrm{d} t .
$$

The integrand of Equation (2.1) is the magnitude of the velocity of the point $X$ as it moves along the curve $X(t)$. If $\frac{\mathrm{d} X}{\mathrm{~d} t} \neq 0$ then the function $s(t)$ can be inverted to obtain $t(s)$ which allows the reparameterization $X(t(s))=X(s)$. The magnitude of $\frac{\mathrm{d} X}{\mathrm{~d} s}$ is

$$
\left\|\frac{\mathrm{d} X(t(s))}{\mathrm{d} s}\right\|=\frac{\mathrm{d} t}{\mathrm{~d} s} \frac{\mathrm{d} s}{\mathrm{~d} t}=1 .
$$

Now, we will use the unit speed form $X(s)$ to define the Frenet frame and the Frenet equations of the curve. And so, we will give interpretation of these results in terms of the general parameter $t$.

The Frenet frame of $X(s)$ is the set of unit vectors, $E, T, N$ and $B$ defined in the following way. The first vector, $E$ is directed along the radius of the hyperquadric and is given by

$$
E=X(s) .
$$

Note that $\|X(s)\|=1$ and $E$ is a spacelike vector. The second vector, $T$, is tangent to $X(s)$ and to $\mathbb{S}_{1}^{3}$. It is obtained by

$$
T=\frac{\mathrm{d} X}{\mathrm{~d} s}
$$

and is a spacelike vector. So, the curve $X(s)$ in $\mathbb{S}_{1}^{3}$ is a spacelike curve. On the other hand, since $T$ is a unit spacelike vector, its derivative $\frac{\mathrm{d} T}{\mathrm{~d} s}$ will be normal to $T$. So, $\frac{\mathrm{d} T}{\mathrm{~d} s}$ will have a component along $E$ given by $\left\langle\frac{\mathrm{d} T}{\mathrm{~d} s}, E\right\rangle$ which we compute by expanding the identity $\frac{\mathrm{d}}{\mathrm{d} s}\langle T, E\rangle=0$ and we get

$$
\left\langle\frac{\mathrm{d} T}{\mathrm{~d} s}, E\right\rangle=-\langle T, T\rangle=-1 .
$$

The remaining component of $\frac{\mathrm{d} T}{\mathrm{~d} s}$ orthogonal to both $E$ and $T$ is chosen as the direction of the unit timelike vector $N$, so we have

$$
N=\frac{\frac{\mathrm{d} T}{\mathrm{~d} s}+E}{\left\|\frac{\mathrm{d} T}{\mathrm{~d} s}+E\right\|} .
$$

Here, we define the function $\kappa_{g}=\left\|\frac{\mathrm{d} T}{\mathrm{~d} s}+E\right\|$, which measures the bend of $X(s)$ out of the $E-T$ plane, to be the geodesic curvature of $X(s)$.

The remaining vector $B$ of the Frenet Frame is obtained by commuting the component of $\frac{\mathrm{d} N}{\mathrm{~d} s}$ which is not along either $E$ or $T$ and choose the direction of $B$ along this component such that the frame taken in the order $T, N, B, E$ has positive orientation. The fact that the component of $\frac{\mathrm{d} N}{\mathrm{~d} s}$ in the direction $E$ is zero is obtained by expanding the identity

$$
\begin{aligned}
& \frac{\mathrm{d}}{\mathrm{d} s}(\langle N, E\rangle)=0, \\
& \left\langle\frac{\mathrm{d} N}{\mathrm{~d} s}, E\right\rangle=-\langle N, T\rangle=0 .
\end{aligned}
$$

On the other hand, by expanding $\frac{\mathrm{d}}{\mathrm{ds}}\langle N, T\rangle=0$ we obtain

$$
\begin{aligned}
\left\langle\frac{\mathrm{d} N}{\mathrm{~d} s}, T\right\rangle & =-\left\langle N, \frac{\mathrm{d} T}{\mathrm{~d} s}\right\rangle=-\left\langle N, \kappa_{g} N-E\right\rangle \\
& =-\kappa_{g}\langle N, N\rangle+\langle N, E\rangle=\kappa_{g} .
\end{aligned}
$$

Therefore we find that the component of $\frac{\mathrm{d} T}{\mathrm{~d} s}$ along $T$ is $\kappa_{g}$. Finally we see that $B$ is given by

$$
B=\sigma \frac{\frac{\mathrm{d} N}{\mathrm{~d} s}-\kappa_{g} T}{\left\|\frac{\mathrm{d} N}{\mathrm{~d} s}-\kappa_{g} T\right\|} .
$$

The function $\tau_{g}=\left\|\frac{\mathrm{d} N}{\mathrm{~d} s}-\kappa_{g} T\right\|$ is defined as the geodesic torsion of $X(s)$. The coefficient $\sigma$ is chosen as either +1 or -1 to ensure that the determinant of the matrix $[T, N, B, E]$ is +1 , that is so that the Frenet frame has positive orientation. The vector $E$ has been choosen as the last member of the frame for several reasons, the primary one being that it is convenient to visu- 
alize the 3-dimensional surface of the hyperquadric locally as the 3-dimensional Lorentzian space of its tangent hyperplane. The vectors $T, N$ and $B$ lie in this space and are analogous to the tangent, normal and binormal vectors of a space curve in three dimensions. In this way the geodesic curvature and torsion functions $\kappa_{g}$ and $\tau_{g}$ are seen to be analogous to the curvature and torsion of a space curve.

Then we have the following proposition.

Proposition 2.1. Let $X(s)$ be a spacelike curve in de-Sitter space $\mathbb{S}_{1}^{3}$. Then the Frenet equations are

$$
\begin{aligned}
\frac{\mathrm{d} T}{\mathrm{~d} s} & =\kappa_{g} N-E \\
\frac{\mathrm{d} N}{\mathrm{~d} s} & =\kappa_{g} T+\sigma \tau_{g} B \\
\frac{\mathrm{d} B}{\mathrm{~d} s} & =\tau_{g} N \\
\frac{\mathrm{d} E}{\mathrm{~d} s} & =T .
\end{aligned}
$$

These equations may be viewed as a set of 16 linear first-order differential equations in the components of $T$, $N, B$ and $E$ which, when the coefficients $\kappa_{g}(s)$ and $\tau_{g}(s)$ are specified functions of $s$, can be solved to determine the curve $E=X(s)$ in $\mathbb{S}_{1}^{3}$. Thus the geodesic curvature and torsion functions, $\kappa_{g}(s)$ and $\tau_{g}(s)$, of $X(s)$ define it completely.

\section{The Local Canonical Form}

The local properties of a hyperquadrical curve $X(s)$ in the vicinity of a reference point $s=s_{0}$ can be obtained by computing the series expansion of $X(s)$ in the Frenet frame of the reference point $X\left(s_{0}\right)$. This form of $X(s)$ is termed the local canonical form by Do Carmo [7].

We choose the coordinate directions of the 4-dimensional Lorentzian space $\mathbb{R}_{1}^{4}$ containing $\mathbb{S}_{1}^{3}$, denoted by $e_{1}, e_{2}, e_{3}, e_{4}$, where $e_{i}$ has a 1 in the $\mathrm{i}$-th coordinate position and zeros elsewhere, so that they align with the Frenet frame $T\left(s_{0}\right), \quad N\left(s_{0}\right), B\left(s_{0}\right)$ and $E\left(s_{0}\right)$ of the reference point $X\left(s_{0}\right)$. Computing the derivatives of $X(s)$ to the third order we have

$$
\begin{aligned}
& X(s)=E, \\
& \frac{\mathrm{d} X}{\mathrm{~d} s}=T, \\
& \frac{\mathrm{d}^{2} X}{\mathrm{~d} s^{2}}=\frac{\mathrm{d} T}{\mathrm{~d} s}=\kappa_{g} N-E, \\
& \frac{\mathrm{d}^{3} X}{\mathrm{~d} s^{3}}=\left(\kappa_{g}^{2}-1\right) T+\frac{\mathrm{d} \kappa}{\mathrm{d} s} N+\sigma \kappa_{g} \tau_{g} B .
\end{aligned}
$$

These expressions lead to the Taylor series expansion of $X(s)$ in the vicinity of the reference position $s=s_{0}$.
For convenience, we denote the reference position as $s_{0}=0$ and obtain

$$
\begin{aligned}
X(s) & =X(0)+\frac{1}{1 !} X^{\prime}(0) s+\frac{1}{2 !} X^{\prime \prime}(0) s^{2}+\frac{1}{3 !} X^{\prime \prime \prime}(0) s^{3} \\
& =\left[\begin{array}{l}
1 \\
0 \\
0 \\
0
\end{array}\right]+\left[\begin{array}{l}
0 \\
1 \\
0 \\
0
\end{array}\right] s+\frac{1}{2}\left[\begin{array}{c}
-1 \\
0 \\
0 \\
\kappa_{g}
\end{array}\right] s^{2}+\frac{1}{6}\left[\begin{array}{c}
0 \\
\kappa_{g}^{2}-1 \\
\sigma \kappa_{g} \tau_{g} \\
\frac{\mathrm{d} \kappa_{g}}{\mathrm{~d} s}
\end{array}\right] s^{3} \\
& =\left[\begin{array}{l}
1 \\
0 \\
0 \\
0
\end{array}\right]+\left[\begin{array}{l}
0 \\
1 \\
0 \\
0
\end{array}\right] s+\left[\begin{array}{c}
-1 \\
0 \\
0 \\
\kappa_{g_{0}}
\end{array}\right] \frac{s^{2}}{2}+\left[\begin{array}{c}
0 \\
\kappa_{g_{0}}^{2}-1 \\
\sigma \kappa_{g_{0}} \tau_{g_{0}} \\
\kappa_{g_{1}}
\end{array}\right] \frac{s^{3}}{6}
\end{aligned}
$$

where $\kappa_{g_{0}}$ and $\tau_{g_{0}}$ are the values of the curvature and torsion of $X(s)$ and $\kappa_{g_{1}}$ is the value of $\frac{\mathrm{d} \kappa_{g}}{\mathrm{~d} s}$, all evaluated at the reference position $s=0$. Equation (3.1) allows a description of the shape of $X(s)$ to various of approximation, for example, to the zeroth order $X(s)$ is simply the point $X(0)$, to the first order it is approximated by the tangent vector $T$. We see that $\kappa_{g_{0}}$ defines the shape of $X(s)$ to the second order which defines the amount that it bends away from the $E-T$ plane. The parameter $\tau_{g_{0}}$ defines the amount that $X(s)$ bends out of the $E-T-N$ subspace. To the second order, $X(s)$ is approximated by its osculating circle which has the radius $\rho$ given by

$$
\rho=\frac{1}{\gamma}=\frac{1}{\left|\left(-\kappa_{g}^{2}+1\right)\right|^{1 / 2}} .
$$

The function $\gamma=\left(-\kappa_{g}^{2}+1\right)^{1 / 2}$ is called the total curvature of $X(s)$. The plane of this circle osculating plane, is defined by the tangent vector $T$ and the unit vector $\mathrm{G}$

$$
G=\frac{\mathrm{d} T / \mathrm{d} s}{\|\mathrm{~d} T / \mathrm{d} s\|}=\frac{\kappa_{g}}{\gamma} N-\frac{1}{\gamma} E .
$$

The rotation of the osculating plane about $T$ is given by $\frac{\mathrm{d} G}{\mathrm{~d} s}$ where

$$
\frac{\mathrm{d} G}{\mathrm{~d} s}=-\gamma T+\frac{\kappa_{g}^{\prime}}{\gamma} \tilde{G}+\frac{\sigma \kappa_{g} \tau_{g}}{\gamma} B
$$

and

$$
\tilde{G}=\left(1+\kappa_{g}^{2}\right) N-\kappa_{g} E .
$$

If $\kappa_{g_{1}}=0$ and $\tau_{g_{0}}=0$ then from (3.3) we see that 
this plane remains instantaneously fixed, i.e.

$$
\frac{\mathrm{d} G}{\mathrm{~d} s}=-\gamma T \text {. }
$$

Furthermore, since $\frac{\mathrm{d} \kappa_{g}}{\mathrm{~d} s}=0$ implies $\frac{\mathrm{d} \gamma}{\mathrm{d} s}=0$ and therefore that the total curvature is constant. A general curve $X(s)$ is approximated to the third order by an osculating sphere $\mathbb{S}_{1}^{2}$. To determine the center $\mathrm{c}$ of this pseudo-sphere we first note that first, second and third derivatives of $X(s)$ lie in the subspace spanned by the three orthogonal unit vectors $T, G$ and $G^{*}$, i.e. we have

$$
\begin{aligned}
& \frac{\mathrm{d} X}{\mathrm{~d} s}=T \\
& \frac{\mathrm{d}^{2} X}{\mathrm{~d} s^{2}}=\kappa_{g} N-E=\gamma G
\end{aligned}
$$

and

$$
\frac{\mathrm{d}^{3} X}{\mathrm{~d} s^{3}}=\frac{\mathrm{d}}{\mathrm{d} s}(\gamma G)=-\gamma^{2} T+\frac{\mathrm{d} \gamma}{\mathrm{d} s} G+\beta G^{*}
$$

where

$$
G^{*}=\frac{\kappa_{g}^{\prime} \tilde{G}+\sigma \kappa_{g} \tau_{g} B}{\left\|\kappa_{g}^{\prime} \tilde{G}+\sigma \kappa_{g} \tau_{g} B\right\|}=\frac{1}{\beta}\left(\kappa_{g}^{\prime} \tilde{G}+\sigma \kappa_{g} \tau_{g} B\right)
$$

and

$$
\beta=\left\|\kappa_{g}^{\prime} \tilde{G}+\sigma \kappa_{g} \tau_{g} B\right\|=\sqrt{\left|\kappa_{g}^{2} \tau_{g}^{2}-\left(\kappa_{g}^{\prime}\right)^{2}\right|} .
$$

Here $G^{*}$ is a spacelike vector if $\kappa_{g}^{2} \tau_{g}^{2}-\left(\kappa^{\prime}\right)^{2}>0$, if not, respectively.

Assume that $G^{*}$ is a timelike vector. So, the radius vector, $R=X(s)-c$, of the osculating sphere must have the form

$$
R=X(s)-c=k_{1} T+k_{2} G+k_{3} G^{*}
$$

where $k_{1}, k_{2}, k_{3}$ are constants. These constants are determined by the requirement that $R$ have constant magnitude to the third order. Differentiating $\langle R, R\rangle=$ const. we obtain

$$
\begin{aligned}
& \left\langle\frac{\mathrm{d} X}{\mathrm{~d} s}, X-c\right\rangle=0, \\
& \left\langle\frac{\mathrm{d}^{2} X}{\mathrm{~d} s^{2}}, X-c\right\rangle+\left\langle\frac{\mathrm{d} X}{\mathrm{~d} s}, \frac{\mathrm{d} X}{\mathrm{~d} s}\right\rangle=0, \\
& \left\langle\frac{\mathrm{d}^{3} X}{\mathrm{~d} s^{3}}, X-c\right\rangle+3\left\langle\frac{\mathrm{d}^{2} X}{\mathrm{~d} s^{2}}, \frac{\mathrm{d} X}{\mathrm{~d} s}\right\rangle=0 .
\end{aligned}
$$

So, from these equations we conclude

$$
\left\{\begin{array}{l}
k_{1}=0, \\
k_{2}=-\frac{1}{\gamma}, \\
k_{3}=-\frac{\gamma^{\prime}}{\gamma \beta} .
\end{array}\right.
$$

Hence, we have

$$
\begin{aligned}
X(s)-c & =-\frac{1}{\gamma} G-\frac{\gamma^{\prime}}{\gamma \beta} G^{*} \\
& =-\frac{1}{\gamma} G-\frac{\gamma^{\prime}}{\gamma \beta^{2}}\left(\kappa_{g}^{\prime} \tilde{G}+\sigma \kappa_{g} \tau_{g} B\right) .
\end{aligned}
$$

For those curves with $\tau_{g}=0$ this relation simplifies using (3.1) and (3.3)

$$
X(s)-c=\frac{\kappa_{g}}{\gamma}\left(\frac{\gamma+\gamma \kappa_{g}^{2}-1}{\gamma}\right) N+\left(\frac{1-\gamma^{2} \kappa_{g}^{2}}{\gamma^{2}}\right) E .
$$

So we have the following proposition.

Proposition 3.1. Let $X(s)$ be a spacelike curve with the geodesic curvature $\kappa_{g}$ and the total curvature $\gamma$ in de-Sitter space $\mathbb{S}_{1}^{3}$. Then the radius vector

$R=X(s)-c$ of the osculating sphere $\mathbb{S}_{1}^{2}$ is as in Equation (3.5).

\section{Arbitrary Parameterization}

We now derive the local canonical form of $X(t)$ with respect to the arbitrary parameter $t$. To do this we use the Frenet equations and the fact that

$$
\left\|\frac{\mathrm{d} X(t)}{\mathrm{d} t}\right\|=\left\|\frac{\mathrm{d} X(t)}{\mathrm{d} s} \cdot \frac{\mathrm{d} s}{\mathrm{~d} t}\right\|=\left\|\frac{\mathrm{d} X(t)}{\mathrm{d} s}\right\|\left\|\frac{\mathrm{d} s}{\mathrm{~d} t}\right\|=v .
$$

Computing the derivatives of $X(t)$ to the third order, we obtain

$$
\begin{aligned}
X(t)=E & \\
\frac{\mathrm{d} X(t)}{\mathrm{d} t}= & v T, \\
\frac{\mathrm{d}^{2} X(t)}{\mathrm{d} t^{2}}= & \frac{\mathrm{d} v}{\mathrm{~d} t} T+v^{2} \kappa_{g} N-v^{2} E, \\
\frac{\mathrm{d}^{3} X(t)}{\mathrm{d} t^{3}}= & \left(\frac{\mathrm{d}^{2} v}{\mathrm{~d} t^{2}}+v^{3}\left(\kappa_{g}^{2}-1\right)\right) T \\
& +\left(3 v \frac{\mathrm{d} v}{\mathrm{~d} t} \kappa_{g}+v^{3} \frac{\mathrm{d} \kappa_{g}}{\mathrm{~d} s}\right) N+\sigma v^{3} \kappa_{g} \tau_{g} B-3 v \frac{\mathrm{d} v}{\mathrm{~d} t} E .
\end{aligned}
$$

Thus the local canonical form for $X(t)$ at a reference point $t=0$ becomes, to third order, 


$$
\begin{aligned}
X(t) & =X(0)+\frac{1}{1 !} \frac{\mathrm{d} X}{\mathrm{~d} t}(0) t+\frac{1}{2 !} \frac{\mathrm{d}^{2} X}{\mathrm{~d} t^{2}}(0) t^{2}+\frac{1}{3 !} \frac{\mathrm{d}^{3} X}{\mathrm{~d} t^{3}}(0) t^{3} \\
& =\left[\begin{array}{l}
1 \\
0 \\
0 \\
0
\end{array}\right]+\left[\begin{array}{l}
0 \\
1 \\
0 \\
0
\end{array}\right] t+\left[\begin{array}{c}
-v^{2} \\
\frac{\mathrm{d} v}{\mathrm{~d} t} \\
0 \\
v^{2} \kappa_{g_{0}}
\end{array}\right] \frac{t^{2}}{2}+\left[\begin{array}{c}
-3 v \frac{\mathrm{d} v}{\mathrm{~d} t} \\
\frac{\mathrm{d}^{2} v}{\mathrm{~d} t^{2}}+v^{3}\left(\kappa_{g_{0}}^{2}-1\right) \\
\sigma v^{3} \kappa_{g_{0}} \tau_{g_{0}} \\
3 v \frac{\mathrm{d} v}{\mathrm{~d} t} \kappa_{g_{0}}+v^{3} \frac{\mathrm{d} \kappa_{g_{1}}}{\mathrm{~d} s}
\end{array}\right] \frac{t^{3}}{6} .
\end{aligned}
$$

\section{Formula for Geodesic Curvature and Torsion}

We now compute the vector $\frac{\mathrm{d} X}{\mathrm{~d} t} \wedge \frac{\mathrm{d}^{2} X}{\mathrm{~d} t^{2}} \wedge X$ which in view of Equation (4.1) becomes

$$
\frac{\mathrm{d} X}{\mathrm{~d} t} \wedge \frac{\mathrm{d}^{2} X}{\mathrm{~d} t^{2}} \wedge X=v^{3} \kappa_{g} T \wedge N \wedge E .
$$

Computing the scalar product of (5.1) with itself, we obtain the following equation for the geodesic curvature $\kappa_{g}:$

$$
\kappa_{g}^{2}=\frac{\left\langle\frac{\mathrm{d} X}{\mathrm{~d} t} \wedge \frac{\mathrm{d}^{2} X}{\mathrm{~d} t^{2}} \wedge X, \frac{\mathrm{d} X}{\mathrm{~d} t} \wedge \frac{\mathrm{d}^{2} X}{\mathrm{~d} t^{2}} \wedge X\right\rangle}{\left(\left\langle\frac{\mathrm{d} X}{\mathrm{~d} t}, \frac{\mathrm{d} X}{\mathrm{~d} t}\right\rangle\right)^{3}} .
$$

And a formula for geodesic torsion $\tau_{g}$ can be given as

$$
\tau_{g}=-\frac{\sigma}{\kappa_{g}^{2}} \operatorname{det}\left(E, \frac{\mathrm{d} X}{\mathrm{~d} t}, \frac{\mathrm{d}^{2} X}{\mathrm{~d} t^{2}}, \frac{\mathrm{d}^{3} X}{\mathrm{~d} t^{3}}\right) .
$$

This relation can be also seen in [2].

\section{Conclusion}

This work develops the differential geometry of spacelike curves on de-Sitter space in four-dimensional Lorentzian space. The motivation for this work is the fact that the Euler parameters of spherical displacements can be used to map them to points on $\mathbb{S}_{1}^{3}$.

\section{REFERENCES}

[1] B. O'Neill, "Semi-Riemann Geometry: With Applictions to Relativity," Academic Press, New York, 1983, 469 p.

[2] T. Fusho and S. Izumiya, "Lightlike Surfaces of Spacelike Curves in de Sitter 3-Space," Journal of Geometry, Vol. 88, 2008, pp. 19-29. http://dx.doi.org/10.1007/s00022-007-1944-5

[3] M. Kasedou, "Singularities of Lightcone Gauss Images of Spacelike Hypersurfaces in de Sitter Space," Journal of Geometry, Vol. 94, 2009, pp. 107-121. http://dx.doi.org/10.1007/s00022-009-0001-y

[4] J. M. McCarthy, "The Differential Geometry of Curves in an Image Space of Spherical Kinematics," Mechanism and Machine Theory, Vol. 22, No. 3, 1987, pp. 205-211. http://dx.doi.org/10.1016/0094-114X(87)90003-6

[5] J. M. McCarthy and B. Ravani, "Differential Kinematics of Spherical and Spatial Motions Using Kinematic Mapping," Journal of Applied Mechanics, Vol. 53, No. 1, 1986, pp. 15-22. http://dx.doi.org/10.1115/1.3171705

[6] B. Ravani and B. Roth, "Mappings of Spatial Kinematics," Journal of Mechanisms, Transmissions and Automation in Design, Vol. 106, No. 3, 1984, pp. 341-347. http://dx.doi.org/10.1115/1.3267417

[7] M. P. Do Carmo, "Differential Geometry of Curves and Surfaces," Prentice-Hall, Englewood Cliffs, 1976, 503 p. 